\title{
Costo-efectividad de prácticas en salud pública: revisión bibliográfica de las intervenciones de la Iniciativa Mesoamericana de Salud
}

\author{
Atanacio Valencia-Mendoza, MCES, (I) Laura G Danese-dISantos, MCES, (I) \\ Sandra G Sosa-Rubí, D en Econ,, (1) Belkis Aracena-Genao, MCSS.(2)
}

\author{
Valencia-Mendoza A, Danese-dISantos LG, \\ Sosa-Rubí SG,Aracena-Genao B. \\ Costo-efectividad de prácticas en salud pública: \\ revisión bibliográfica de las intervenciones \\ de la Iniciativa Mesoamericana de Salud. \\ Salud Publica Mex 20I I;53 supl 3:S375-S385.
}

\section{Resumen}

Objetivo. Presentar y analizar información de costo-efectividad de intervenciones propuestas por la Iniciativa Mesoamericana de Salud (IMS) en las áreas de nutrición infantil, inmunizaciones, paludismo, dengue y salud materno-infantil y reproductiva. Material y métodos. Se llevó a cabo una revisión sistemática de la literatura de evaluaciones económicas publicadas entre el año 2000 y agosto 2009 sobre intervenciones en las áreas de la salud mencionadas, en los idiomas inglés y español. Resultados. Las intervenciones en nutrición y de salud materno-infantil mostraron ser altamente costo-efectivas (con rangos menores a US\$200 por año de vida ajustado por discapacidad [AVAD] evitado para nutrición y US\$ 100 para materno-infantil). En dengue sólo se encontró información sobre la aplicación de larvicidas, cuya razón de costo efectividad estimada fue de US\$40.79 a US\$345.06 por AVAD evitado. Respecto al paludismo, las intervenciones estudiadas resultaron costo-efectivas (<US\$150 por AVAD evitado o US\$4000 por muerte evitada en países africanos). Para vacunación contra neumococo y rotavirus, los rangos de costo por AVAD evitado superan un producto interno bruto (PIB) per cápita en la mayoría de los países centroamericanos. Conclusiones. En Mesoamérica persisten importantes rezagos en materia de salud, relacionados con las áreas de nutrición infantil, inmunizaciones, paludismo, dengue y salud
Valencia-Mendoza A, Danese-dISantos LG, Sosa-Rubí SG,Aracena-Genao B.

Cost-effectiveness of public health practices: A literature review of public health interventions from the Mesoamerican Health Initiative.

Salud Publica Mex 20I I;53 suppl 3:S375-S385.

(I) Dirección de Economía de la Salud, Instituto Nacional de Salud Pública. Cuernavaca, Morelos, México.

(2) Centro de Investigación en Sistemas de Salud, Instituto Nacional de Salud Pública. Cuernavaca, Morelos, México.

Fecha de recibido: 12 de enero de 20II - Fecha de aceptado: I4 de junio de 20II

Autor de correspondencia: Laura G. Danese de los Santos. Dirección de Economía de la Salud, Instituto Nacional de Salud Pública. Av. Universidad 655, Cuernavaca, Morelos, 62100 México.

Correo electrónico: laura.danese@correo.insp.mx 
materno-infantil y reproductiva, rezagos sujetos de corregir con intervenciones técnicamente factibles y altamente costo-efectivas.

Palabras clave: políticas públicas de salud; costo-efectividad; salud pública; Mesoamérica that could be addressed by scaling-up technically feasible and cost-effective interventions.

Key words: Public health policy; cost effectiveness; public health; Mesoamerica
$E_{s}^{1}$ Sistema Mesoamericano de Salud Pública (SMSP) se creó con el propósito de traducir en acciones concretas los objetivos de salud del Proyecto Mesoamérica. ${ }^{1}$ Fue creado para dar respuesta eficaz y oportuna a los retos comunes en materia de salud en los estados del sur-sureste de México y los países de Centroamérica, y funcionar como un mecanismo de coordinación y desarrollo de actividades de cooperación multilateral que logre consolidar la cooperación técnica en materia de salud pública. ${ }^{1}$

Existe consenso en que los retos más importantes en materia de salud que requieren atención conjunta en la región se circunscriben a cuatro áreas de intervención, que son: nutrición infantil, salud materno-infantil y reproductiva (SMIR), inmunizaciones y enfermedades transmitidas por vectores, específicamente dengue y paludismo. En general, el SMSP busca disminuir las brechas de cobertura de salud en la región, implementar intervenciones de salud que sean altamente costo-efectivas y fortalecer la capacidad de salud pública regional. ${ }^{1}$

Para dar cumplimiento a los objetivos del SMSP, se llevó a cabo un proceso de planeación estratégica, del que derivó la formación de un grupo de expertos de la región para cada una de las áreas de intervención previamente identificadas. Una de las tareas iniciales de estos grupos fue el desarrollo de un perfil situacional, acompañado de un plan maestro en el que se proponen estrategias de intervención basadas en la elección de prácticas de probada efectividad. La descripción en detalle del perfil situacional y de los planes maestros de cada área puede encontrarse en los artículos de GómezDantés y cols., Rivera y cols., Hernández y cols., SantosPreciado y cols., Rodríguez y cols. y Gómez-Dantés y cols., publicados en este mismo número.

Dados los limitados recursos de que disponen los países de la región para atender las problemáticas de salud, uno de los propósitos del SMSP es implementar intervenciones que, además de ser de probada efectividad, sean costo-efectivas. Para contribuir con este objetivo, en este artículo se presenta una revisión sistemática de la literatura de costo-efectividad de las prácticas que han probado ser efectivas en la atención o prevención de la desnutrición infantil, salud materno- infantil y reproductiva, enfermedades prevenibles por vacunas, dengue y paludismo. A continuación se describen las intervenciones identificadas por los grupos de expertos.

\section{Nutrición}

El grupo de expertos en nutrición identificó que los retos más importantes en la materia en la región mesoamericana son la anemia y desnutrición crónica, asociadas con una alimentación inadecuada en etapas clave del crecimiento. Para combatir dichos problemas nutricionales, los expertos identificaron un grupo de intervenciones ${ }^{2}$ con probada efectividad que incluyen: 1) promover la lactancia materna exclusiva durante los seis primeros meses; 2) aconsejar en prácticas adecuadas de alimentación complementaria, y 3) promover prácticas de higiene. Estas tres intervenciones se enfocan en el cambio de comportamiento, sin provisión de complementos alimenticios. Para combatir deficiencias en micronutrientes, se propuso: 4) suplementar con vitamina $\mathrm{A}$ a niños menores de 5 años; 5 ) en casos de niños menores de dos años con diarrea, suplementar con zinc; 6) proveer micronutrientes en polvo para la alimentación complementaria de niños menores de dos años; 7) suplementar con hierro y ácido fólico a mujeres embarazadas, y 8) fortificar alimentos en todo el país. Además, se propuso para las regiones con alta vulnerabilidad: 9) proveer de alimentos complementarios fortificados a niños de 6 a 23 meses, y 10) dar seguimiento a nivel clínico de los protocolos estandarizados de tratamiento para la malnutrición aguda severa (SAM, por sus siglas en inglés). Finalmente, como intervenciones relacionadas efectivas, se recomienda: 11) promover los programas de transferencias condicionadas (CCT, por sus siglas en inglés) con un componente nutricional, y 12) llevar a cabo acciones para promover drenaje y provisión de agua.

\section{Salud materno-infantil y reproductiva}

El grupo de expertos en salud materno-infantil y reproductiva propuso un plan enfocado en el mejoramiento 
del acceso a servicios de salud de alta calidad y la utilización equitativa de los mismos. ${ }^{3}$ La población objetivo son las mujeres en edad reproductiva (14-49 años) y los recién nacidos, dentro de los grupos de población más pobres, indígenas, además de las poblaciones marginadas de zonas urbanas. Las intervenciones propuestas se dividen en tres áreas:

Salud materna: atención de emergencias obstétricas básicas e intervenciones para mejorar el manejo de emergencias obstétricas relacionadas con infecciones, eclampsia, hemorragia y aborto; creación de conciencia en la comunidad y educación y entrenamiento del personal.

Salud neonatal: atención esencial al recién nacido, atención dirigida al recién nacido de bajo peso y atención de emergencia para recién nacidos con complicaciones; vacuna contra tétanos y tratamiento de septicemia.

Planificación familiar y salud reproductiva: servicios de planificación familiar de calidad dirigida a adolescentes; acceso a consejería y servicios para, por lo menos, seis tipos de métodos anticonceptivos, además de servicios de consejería de anticonceptivos en atención posparto y posaborto.

\section{Paludismo y dengue}

Las estrategias contra el paludismo propuestas por los expertos incluyen: a) vigilancia epidemiológica con búsqueda intensiva de febriles y promoción de la notificación, para lo que se ha considerado el fortalecimiento de la red de notificantes voluntarios en las localidades endémicas con registro de casos en el periodo 2006-2008; b) control de vectores, que incluye el control larvario (mediante control físico de 100\% de los criaderos y control químico selectivo en las localidades y criaderos que, de acuerdo con la evaluación entomológica, lo requieran), así como la prevención del contacto del humano-vector mediante el rociado intradomiciliar y la distribución de mosquiteros impregnados con insecticida de acción residual prolongada; c) tratamiento de casos en pacientes con un diagnóstico confirmado de paludismo, los cuales serán tratados con dosis única mensual (TDUM) durante tres meses, y d) detección y control de brotes en situaciones de emergencia, en las que se ejecutarán acciones intensivas de rociado de viviendas, diagnóstico y tratamiento y búsqueda activa.

Las acciones contra el dengue incluyen: a) vigilancia epidemiológica y entomológica; b) control de vectores mediante la eliminación o control de los criaderos productivos, aplicación de larvicidas, rociado intradomiciliar y espacial (nebulizaciones); c) promoción de participación activa de la comunidad, estrategia fundamental en el control del dengue, ya que muchas de las estrategias previamente señaladas, como la eliminación de criaderos (descacharrización) o el control de criaderos en recipientes de almacenaje de agua potable, requieren de una activa participación de la comunidad; d) diagnóstico y tratamiento oportuno de los pacientes con dengue, con énfasis en los que tienen dengue hemorrágico, para lo que se requiere fortalecer la infraestructura de laboratorio. La vacuna contra dengue se encuentra en desarrollo, por lo que aún no es una realidad como estrategia de salud pública.

\section{Inmunizaciones}

El grupo de expertos en inmunizaciones propuso un conjunto de intervenciones en tres líneas de acción diferentes: 1) estudios piloto para cerrar las brechas de información para la toma de decisiones; 2) fortalecimiento de las políticas de inmunización; y 3) implementación de prácticas basadas en evidencia. Todas estas son acciones sistémicas para aumentar los niveles de cobertura de los programas ampliados de inmunizaciones en los países mesoamericanos. También se consideró muy importante la introducción de vacunación contra rotavirus y el Streptococcus pneumoniae o neumococo. Debido a que la mayor parte de las intervenciones propuestas por este grupo son a nivel sistémico, para las cuales se desconoce su efectividad y costo-efectividad, sólo se presentan resultados de la revisión de la literatura de costo-efectividad para las vacunas contra el rotavirus y el neumococo.

\section{Material y métodos}

Se realizó una búsqueda exhaustiva y sistemática de la literatura de costo-efectividad de las intervenciones propuestas por los expertos de las cuatro áreas, en la cual se tomaron en cuenta artículos publicados entre enero de 2000 y agosto de 2009, en los idiomas inglés y español. Se exploraron las bases de datos PubMed, The Cochrane Library, ISI Web of Science, Econlit, ERIC, Medline, Lilacs, además de páginas electrónicas especializadas en cada materia de estudio. Para nutrición se incluyó Food and Nutrition Library; para SMIR se exploraron Medicus y la Biblioteca de Salud Reproductiva de la Organización Mundial de la Salud (OMS); para paludismo y malaria se revisaron el Instituto Colombiano de Medicina Tropical, la Asociación Americana de Control de Mosquitos y la Asociación Americana de Salud Pública, además de artículos inéditos, encontrados a través de Google Scholar. Adicionalmente, se revisó la base de datos del proyecto de la OMS para seleccionar intervenciones 
costo-efectivas (WHO-CHOICE CHOosing Interventions that are Cost-Effective). ${ }^{4}$ También se contactaron expertos en las diferentes áreas, quienes enviaron textos inéditos e informes técnicos relevantes. Finalmente, se identificaron otros documentos de interés por medio de la revisión de la lista de citas de los artículos seleccionados para su revisión en extenso.

En la búsqueda se emplearon palabras clave que aparecieran en el título o resumen de los artículos. Los términos de búsqueda compuestos por las palabras clave fueron revisados por los expertos en los diferentes pilares y se presentan en el cuadro I. Los criterios de búsqueda para el caso SMIR se dieron en dos niveles: de intervención y de paquetes de intervenciones. Para el área de nutrición, primeramente se llevó a cabo una búsqueda inicial por programas que incluyeran intervenciones nutricionales y luego por intervenciones específicas.

Debido a que la razón de costo-efectividad de vacunación contra rotavirus y contra neumococo depende, en gran medida, de las situaciones epidemiológicas locales en ausencia de vacunación, como incidencia, letalidad y prevalencia de los serotipos circulantes contenidos en las vacunas; de los costos locales de la enfermedad y del precio por dosis de la vacuna, los resultados aquí presentados corresponden únicamente a los estudios llevados a cabo en contextos de países latinoamericanos.
La selección de artículos se llevó a cabo en tres fases: primero se descartó por títulos, después por resúmenes y finalmente se analizó el documento completo. Se excluyeron los estudios que fueran exclusivos de efectividad o exclusivos de costos y se aceptaron estudios publicados antes del año 2000, en el caso de intervenciones donde no existieran publicaciones más recientes.

Con el propósito de valorar la calidad metodológica de los artículos, así como conocer el contexto en el cual los estudios fueron hechos y los supuestos adoptados, se desarrolló una matriz de extracción de información en Excel. Entre otras cosas, esta matriz recogió datos de la región donde se realizó el estudio; las intervenciones estudiadas; el tipo de costos e información de efectividad que utiliza el estudio; la perspectiva del análisis; los resultados, y las conclusiones del estudio. Para el análisis de los datos, todos los resultados fueron transformados a dólares de Estados Unidos de 2008, utilizando el promedio del índice de precios al consumidor que reporta el U.S. Department of Labor.

\section{Resultados}

En el caso de nutrición se revisaron en extenso 36 estudios de los cuales se le dio prioridad a los trabajos en los que se analiza algún país de América Latina. ${ }^{5-13}$ También se incorporó la información del ejercicio propuesto por

\section{Cuadro I \\ Palabras clave de búsqueda}

Palabras clave comunes para los cuatro pilares:

(Cost-effectiveness OR economic evaluation OR cost effectiveness OR cost analysis OR cost benefit OR cost-benefit OR cost utility OR cost effect OR economic impact)

Palabras clave específicas de cada pilar:

(malnutrition OR undernutrition OR nutritional deficiency OR weight OR weight for height OR height for age OR birth weight OR stunting OR anemia OR stunted OR diet) AND (Breastfeeding OR Complementary Feeding OR complementary food OR Supplementary Feeding OR Dietary Supplement OR Dietary Supplementation OR Food Supplementation OR Food Supplements OR Neutraceuticals OR fortified food OR Enriched foods OR zinc OR iron OR vitamin A OR retinol OR micronutrient OR dietary intake OR sprinkles OR interventions OR social programs OR public health programs).

(Vaccine OR Vaccination OR conjugate vaccine OR PCV7 OR Heptavalent Vaccine OR Immunization OR Protection OR Schedule) AND (streptococcus Pneumoniae OR pneumococcus OR pneumococcal infections OR pneumococcal meningitis OR pneumococcal otitis OR pneumococcal pneumonia OR pneumococcal sepsis OR pneumococcal bacteria OR pneumococcal)

(Maternal OR perinatal OR reintervention OR prenatal care OR antenatal care OR neonatal care OR emergency prenatal OR obstetric care OR delivery care OR infant care OR emergency obstetric OR interventions OR package OR strategies OR neonatal health OR maternal baby OR maternal health).

(Malaria OR plasmodium falciparum OR plasmodium vivax OR Insecticide-treated nets OR permethrin OR Larvivorous fish OR malaria control OR Temephos OR In-house residual spraying $O R$ bednets $O R$ antimalarials drugs $O R$ antimalarial treatment $O R$ chloroquine ORcommunity participation).

(Dengue OR dengue fever OR dengue hemorrhagic OR Aedes aegypti OR Aedes albopictus) AND (vector control OR larval control OR larviciding campaigns OR insecticide OR pupal OR ovitraps OR temephos OR closed houses OR Pre-seasonal focal treatment OR diagnostic test OR mosquito control OR biological control OR chemical control OR abate OR community practices OR Bacillus thuringiensis OR foci OR copepod OR control vectorial OR container breeding OR lethal ovitrap OR pupa survey OR cis-Perrnethrin OR larvivorous fish OR jar covers OR driving forces) 
la OMS de costo-efectividad de intervenciones nutricionales para dicha región.

Para el caso de SMIR, la literatura más relevante se centró en los paquetes de intervenciones. Se seleccionaron 23 estudios para su revisión. Dentro de las contribuciones más importantes destacan la investigación del Proyecto Control de Enfermedades de Prioridades (DSPP) ${ }^{14}$ y tres revisiones de costo-efectividad de intervenciones en salud materno neonatal. ${ }^{15-17}$ Finalmente, también destaca la información del ejercicio propuesto por la OMS de costo-efectividad de paquetes diseñados para la salud materno y neonatal en la región de América Latina.

En el área de enfermedades transmitidas por vector, se revisaron 23 artículos en extenso, de los cuales 18 correspondieron a intervenciones contra paludismo y cinco contra dengue. Sin embargo, para las intervenciones que se analizaron, sólo dos artículos de dengue reportaron el costo por año de vida ajustado por discapacidad (AVAD) ${ }^{18,19}$ y ninguno reportó el costo por muerte evitada. Para el caso de paludismo, siete reportaron el costo por $\operatorname{AVAD}^{20-26}$ y nueve lo hicieron por muerte evitada. ${ }^{20-26,27,28}$

Para el caso de vacunación, la revisión constó de 10 artículos, de los cuales tres correspondieron a vacunación contra neumococo conjugada heptavalente, ${ }^{29-31}$ seis a vacunación monovalente contra rotavirus, ${ }^{32-37}$ y uno a vacunación pentavalente contra rotavirus. ${ }^{38}$ Los resultados son reportados tanto en términos de costo por AVAD evitado, costo por año de vida ganado, como en costo por muerte evitada.

En las figuras 1-4 se presentan los hallazgos de costo-efectividad para las intervenciones en cada una de las cuatro áreas estudiadas. En los gráficos se presentan tanto estimaciones puntuales como barras que representan los rangos de costo-efectividad cuando son reportados. Estos rangos pueden representar tanto rangos de incertidumbre dentro de un mismo estudio, como variación en estimaciones entre distintos estudios.

\section{Intervenciones en nutrición}

Las intervenciones consideradas más costo-efectivas son las enfocadas a consejería o cambios en comportamiento. Para la promoción de higiene, promoción de lactancia materna y consejería en prácticas de alimentación complementaria, se han estimado razones de costo-efectividad menores a \$15 por AVAD evitado (figura 1). ${ }^{7,9,39}$

Fortificar de forma universal alimentos con vitamina Ay zinc, así como suplementar a niños con vitami-

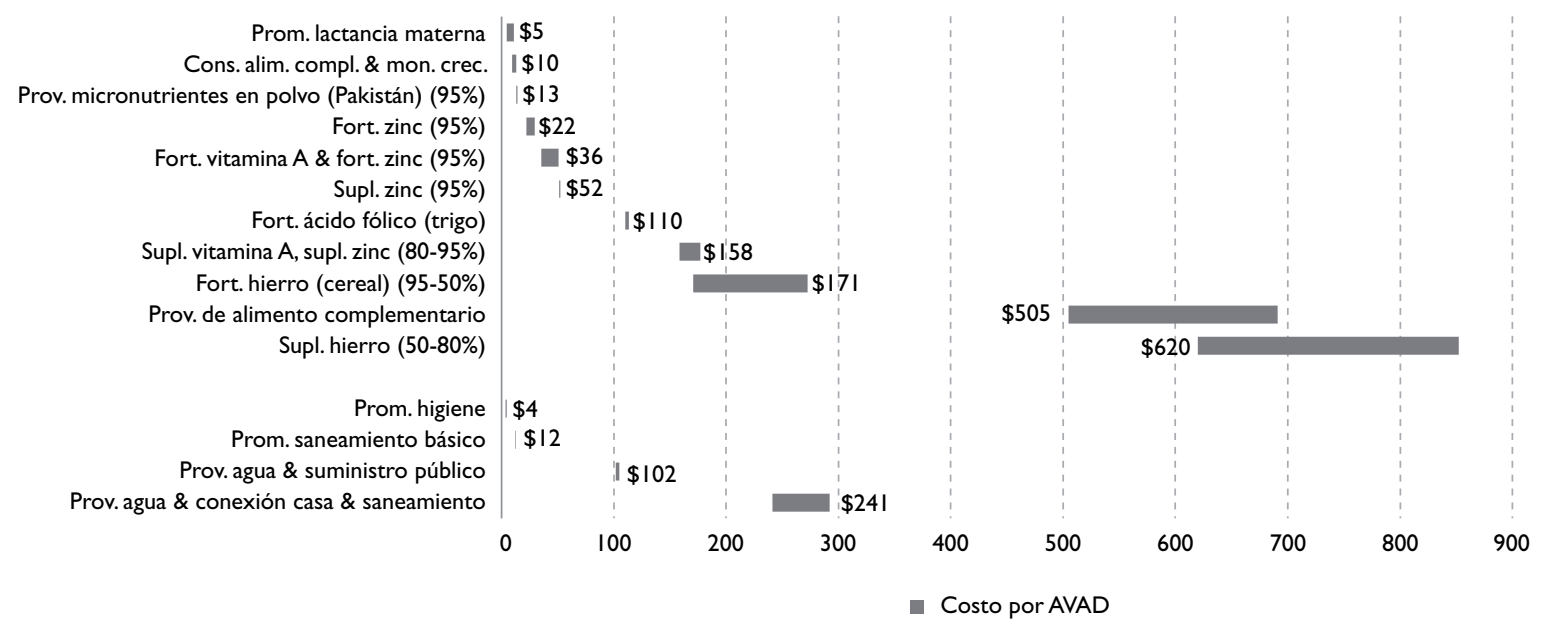

Notas:

Entre paréntesis se definen los porcentajes de las coberturas de la intervención.

Cons. Alim. Compl. \& Mon. Crec.- consejería en alimentación complementaria y monitoreo del crecimiento.

Datos para diferentes países de América Latina.

Prom.-promoción

Prov. - provisión

Fort. - fortificación

Supl. - suplementación

Fuente: Elaboración propia a partir de la revisión de la literatura. Cuernavaca, Morelos, México, 2010.

\section{Figura I. Costo-efectividAd de INTERVEnCiONeS EN NUTRICIÓN}


na A, suplementar a niños con zinc en casos de diarrea, suplementar con ácido fólico a mujeres embarazadas, o proveer micronutrientes en polvo, son estrategias cuya razón de costo-efectividad resulta menor a \$200 por AVAD evitado (figura 1). En el caso de vitamina A y zinc, fortificar tiende a ser más costo-efectivo que suplementar; sin embargo, estas intervenciones no pueden considerarse sustitutas porque los alimentos fortificados de forma universal no cubren las necesidades de micronutrientes de ciertas poblaciones, como las de los niños con deficiencias. ${ }^{40,41}$

Suplementar con hierro a mujeres embarazadas y proveer alimentos complementarios fortificados son de las intervenciones más caras; sin embargo, por su alta efectividad, se mantienen a una razón de costoefectividad por debajo de los $\$ 900$ por AVAD evitado (figura 1). Además, estas intervenciones podrían ser más atractivas desde el punto de vista de la salud pública, cuando se considera que proveen beneficios que no son capturados a través de las estimaciones de AVAD, como son el mayor desarrollo cognitivo a largo plazo. Finalmente, se encontró que la provisión de agua limpia y acceso a saneamiento tiene una razón costo-efectividad menor a $\$ 300$ por AVAD evitado.

No se encontraron datos de costo-efectividad para tratamiento clínico de SAM, ni para el caso de intervenciones de nutrición ofrecidas en el contexto de programas de transferencias condicionadas.

\section{Salud materno-infantil y reproductiva}

En países en desarrollo de África se han encontrado razones de costo-efectividad incremental de ocho dólares por AVAD evitado por el uso de toxoide tetánico en el embarazo; ${ }^{42}$ mientras que, en Asia, dicha razón ha sido de aproximadamente $\$ 3.61(95 \% \mathrm{CI}=\$ 2.43-6.39) .{ }^{43}$

En Estados Unidos se encontró que el uso de cualquier método anticonceptivo es superior a la opción "sin método" en términos de costo-efectividad. Entre los métodos más costo-efectivos se encuentra el dispositivo intrauterino, la vasectomía y el LNG-IUS 20 (sistema intrauterino con liberación de levonorgestrel). Su costo es de $\$ 6.7, \$ 7.4$ y $\$ 9.6$, respectivamente, por tasa anual al evitar un embarazo durante cinco años. ${ }^{44}$

Investigaciones recientes han puesto de manifiesto que la implementación de paquetes de intervenciones para la salud materna y neonatal han mostrado razones costo-efectivas mejores que las intervenciones implementadas de manera individual. ${ }^{42}$ Con el fin de ampliar la cobertura de paquetes de intervenciones para la atención del embarazo, parto y atención neonatal, se utilizó un ejercicio de 2005 que aplica métodos estandarizados propuestos por la OMS. ${ }^{42,45}$ Este ejercicio analiza los datos de costos-efectividad del proyecto WHO-CHOICE. El análisis considera estimaciones de costo-efectividad de paquetes compuestos a partir de 21 intervenciones. Las intervenciones contenidas en los paquetes pueden ser provistas en diferentes niveles de atención: primer nivel de atención, referencia de casos complicados, atención prenatal y atención del recién nacido (para la madre y el bebé) en clínicas y hospitales, así como la atención comunitaria para los recién nacidos. La lista de intervenciones se refieren a las necesarias en los periodos prenatal, intraparto, puerperio y recién nacido. Los efectos se midieron en AVAD evitados. El estudio calculó costos, efectos y rentabilidad de 300 diferentes combinaciones de intervenciones analizadas por la OMS, de acuerdo con el perfil epidemiológico de las subregiones.

En este artículo se resumen y ordenan las intervenciones más rentables en América Latina, en general, y para el grupo de países con las tasas más altas de mortalidad infantil (Bolivia, Ecuador, Guatemala, Haití, Nicaragua y Perú). En la figura 2 se muestran los resultados de costo por AVAD evitado para aquellas intervenciones que no fueron dominadas, es decir, para las que no hubo otra intervención que al mismo tiempo resultó más efectiva y menos costosa. En las dos regiones mencionadas, las intervenciones para los recién nacidos, implementadas en el ámbito comunitario, son altamente costo-efectivas, en especial, el tratamiento de la neumonía neonatal y el paquete comunitario para la atención del recién nacido. ${ }^{45}$

Las intervenciones más costo-efectivas contenidas en paquetes en América Latina son el uso de la vacuna toxoide tetánico, la detección bacteriuria asintomática, el parto normal por personal capacitado, la detección de preeclampsia, la gestión de parto obstruido, la atención esencial al recién nacido, la atención de septicemia materna, atención materna y cuidado del recién nacido calificados, el tratamiento de preeclampsia/eclampsia grave (PEE), el tratamiento para la hemorragia posparto primaria y de referencia (figura 2). Para la región de América Latina, siete de las 10 combinaciones de intervenciones permanecen por debajo de los $\$ 100$ por AVAD evitado.

Entre países de América Latina con altas tasas de mortalidad materna e infantil, el conjunto de paquetes que resulta con mejores resultados de costo-efectividad cambia ligeramente. Este conjunto está conformado por la atención de recién nacidos prematuros con esteroides, uso de antibióticos RPM, manejo de parto obstruido, atención esencial al recién nacido, atención de septicemia materna referida, atención calificada de la madre y el recién nacido, tratamiento de preeclampsia/ eclampsia grave, tratamiento para la hemorragia posparto primaria referida (figura 2). En este caso, siete de las ocho 


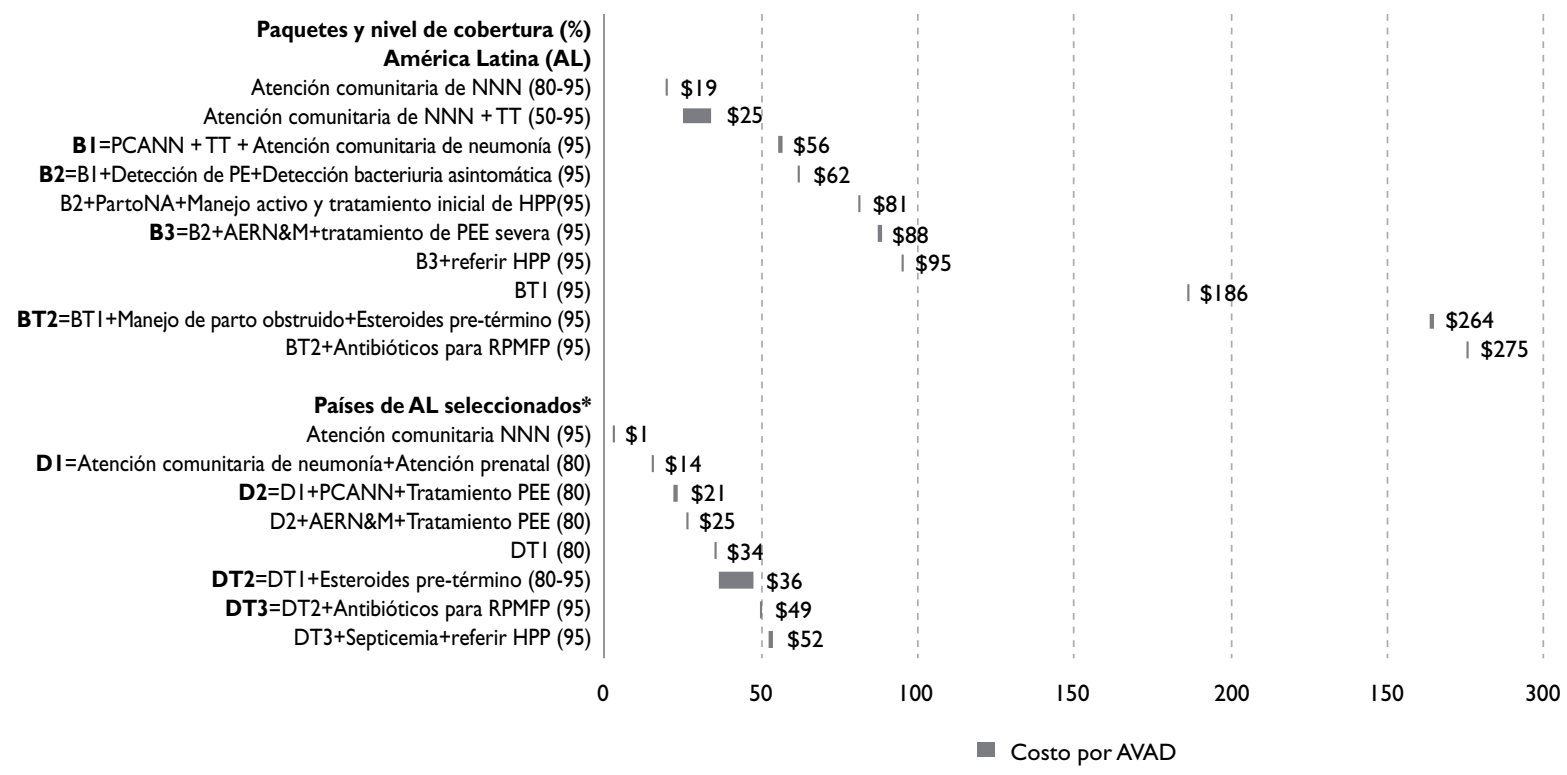

Notas:

NNN: Neumonia neonatal

$\mathrm{TT}$ :Toxoide tetánico

PCANN: Paquete comunitario de atención neonatal

PE: Preeclampsia

PartoNA: Parto normal asistido por personal capacitado

HPP: Hemorragia posparto

PEE: Preeclampsia/eclampsia

AERN\&M:Atención especializada de recién nacido y la madre

RPMF: Ruptura prematura de membranas fetales pre-término

$\mathrm{mBPN}$ : Muy bajo peso al nacer

Fuente: Elaboración a partir de los resultados del proyecto WHO-

CHOICE. Cuernavaca, Morelos, México, 2010.

\footnotetext{
* Los países seleccionados incluyen Ecuador, Perú, Guatemala, Haití, Nicaragua y Bolivia.

BTI: Paquete comunitario de atención neonatal + Atención comunitaria de neumonía + Atención prenatal + AERN\&M + Tratamiento de PEE severa + referir HPP + Septicemia + Atención neonatal de emergencia DTI:Atención comunitaria de neumonía + Atención prenatal + Paquete comunitario de atención neonatal + AERN\&M + Tratamiento de PEE + Manejo de parto obstruido + Atención neonatal de emergencia AVAD:Año de vida ajustado por discapacidad
}

Figura 2. COSTO-EFECTIVIDAD DE INTERVENCIONES EN ATENCIÓN MATERNO-INFANTIL

intervenciones presentan una razón de costo-efectividad por debajo de los $\$ 50$ por AVAD evitado, mientras que la octava intervención apenas rebasa esta cifra.

\section{Paludismo y dengue}

En la figura 3 se muestran los resultados de la evidencia disponible sobre costo-efectividad de intervenciones de dengue (D-) y paludismo (P-). Para el caso de dengue existe muy poca evidencia de costo-efectividad. Aunque la vacunación no es todavía una alternativa de salud pública, podría ser la intervención más costo-efectiva, una vez desarrollada y aprobada para su uso. Con base en el análisis de un modelo matemático, Sheppard y colaboradores estiman que el costo por AVAD evitado de vacunar a niños de hasta 15 meses de edad estaría en un rango de $\$ 21.97$ a $\$ 64.67$, esto bajo el supuesto de que la vacuna pediátrica contra dengue llegara a ser segura y efectiva..$^{19}$ Por otro lado, para el caso de control vectorial, se encontró que la aplicación de larvicidas resulta en una razón de costo-efectividad de $\$ 40.79$ a $\$ 345.06$ por AVAD evitado.

Los resultados de paludismo en la figura 3 indican que la estrategia más costo-efectiva es el tratamiento de pacientes infectados con sulfadoxine-pyrimethamine, con rango de costo por AVAD evitado de \$3.95-\$11.96, y de \$107.01-\$321.57 por muerte evitada. La siguiente intervención que mostró ser altamente costo-efectiva fue la distribución gratuita de mosquiteros impregnados con insecticida de acción residual prolongada en grupos de alto riesgo con $\$ 14.33-\$ 48.51$ por AVAD evitado y \$482.86-\$1 597.41 por muerte evitada.

El control físico de criaderos, actividad que tiene un importante componente de participación comunitaria, 


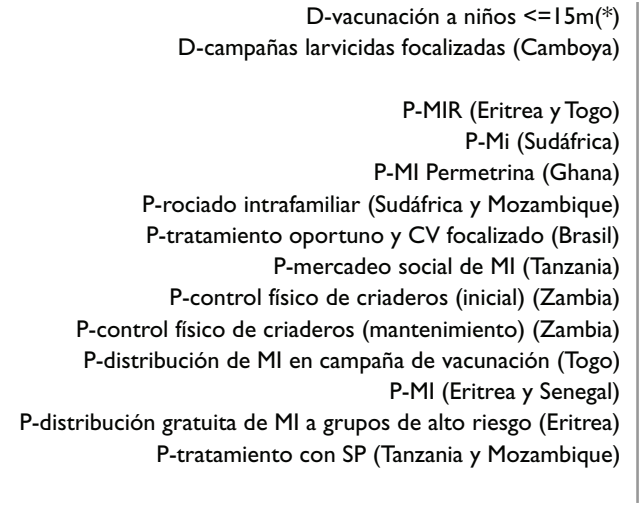

0

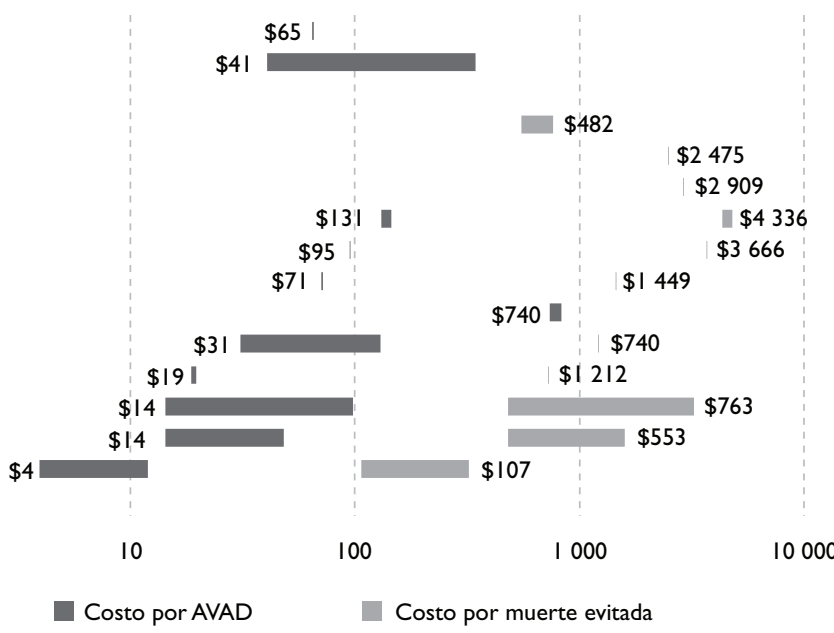

* Modelación para el contexto del Sureste de Asia, asumiendo la introducción de una vacuna con eficacia de $95 \%$, inmunización completa de dos dosis a un precio de US\$0.05

Notas: CV: Control vectorial; MIR: mosquiteros impregnados con insecticida de acción residual; MI: Mosquiteros impregnados; SP: sulfadoxine-pyrimethamine. La gráfica se muestra en escala logarítmica para fines visuales

Fuente: Elaboración propia a partir de la revisión de la literatura. Cuernavaca, Morelos, México, 20I0

Figura 3. Costo-efectividad de intervenciones para el control de paludismo (P-) y dengue (D-)

y la impregnación convencional de mosquiteros fueron las otras intervenciones que se aproximaron al rango de valores considerado en este estudio para definir las intervenciones altamente costo-efectivas. La intervención de control físico de criaderos tuvo un rango de costo por AVAD evitado de \$31.08-\$129.97 y un costo promedio por muerte evitada de $\$ 1212.14$, y los costos reportados por la bibliografía para la intervención de impregnación de mosquiteros oscilaron entre $\$ 14.33$ y $\$ 98.11$ por AVAD evitado y $\$ 482.86-\$ 3225.68$ por muerte evitada.

\section{Inmunizaciones}

Se encontraron tres publicaciones que reportan resultados sobre vacuna heptavalente contra neumococo, de los cuales, uno corresponde a un análisis hecho en un grupo de países elegibles para recibir apoyo de la Global Alliance for Vaccines \& Immunization (GABI), entre los que figuran algunos de América Latina. Los otros dos artículos corresponden a análisis multipaís en América Latina. Se encontró que el rango de las estimaciones de costo-efectividad estuvo entre \$74 y $\$ 5735$ por AVAD evitado y entre \$2 200 y \$175 000 por muerte evitada (figura 4).

En el caso de la vacunación con vacuna monovalente contra rotavirus se encontró que las estimaciones de costo-efectividad oscilaron entre \$269 y \$11 261 por AVAD evitado, $\$ 810$ y $\$ 1174$ por año de vida ganado, y entre $\$ 8972$ y $\$ 53443$ por muerte evitada. En el caso de la vacuna pentavalente contra rotavirus, la razón de costo-efectividad osciló entre \$1 719 y \$4 283 por año de vida ganado y $\$ 122058$ por muerte evitada (figura 4).

\section{Discusión}

En Mesoamérica aún persisten problemas de salud en las poblaciones más vulnerables que, de acuerdo con los resultados mostrados en este artículo, tienen un amplio margen de oportunidad para mejorarse con intervenciones técnicamente factibles y altamente costo-efectivas.

En el caso de la nutrición, la mayoría de las intervenciones descritas presentan razones de costo-efectividad menores a los $\$ 200$ por AVAD evitado, mientras que en el caso de la salud materno-infantil, la mayoría de las combinaciones de intervenciones presentan una relación de costo-efectividad menor a \$100. La Comisión sobre Macroeconomía y Salud de la OMS sugiere que las intervenciones pueden considerarse como altamente costo-efectivas, si su costo por AVAD es menor a una vez el PIB per cápita; se consideran potencialmente costoefectivas, si su razón de costo por AVAD evitado está entre una y tres veces el PIB per cápita y se consideran no costo-efectivas cuando el costo por AVAD evitado es mayor a tres veces el PIB per cápita de un país. De acuerdo con estos criterios, y tomando en cuenta las cifras 


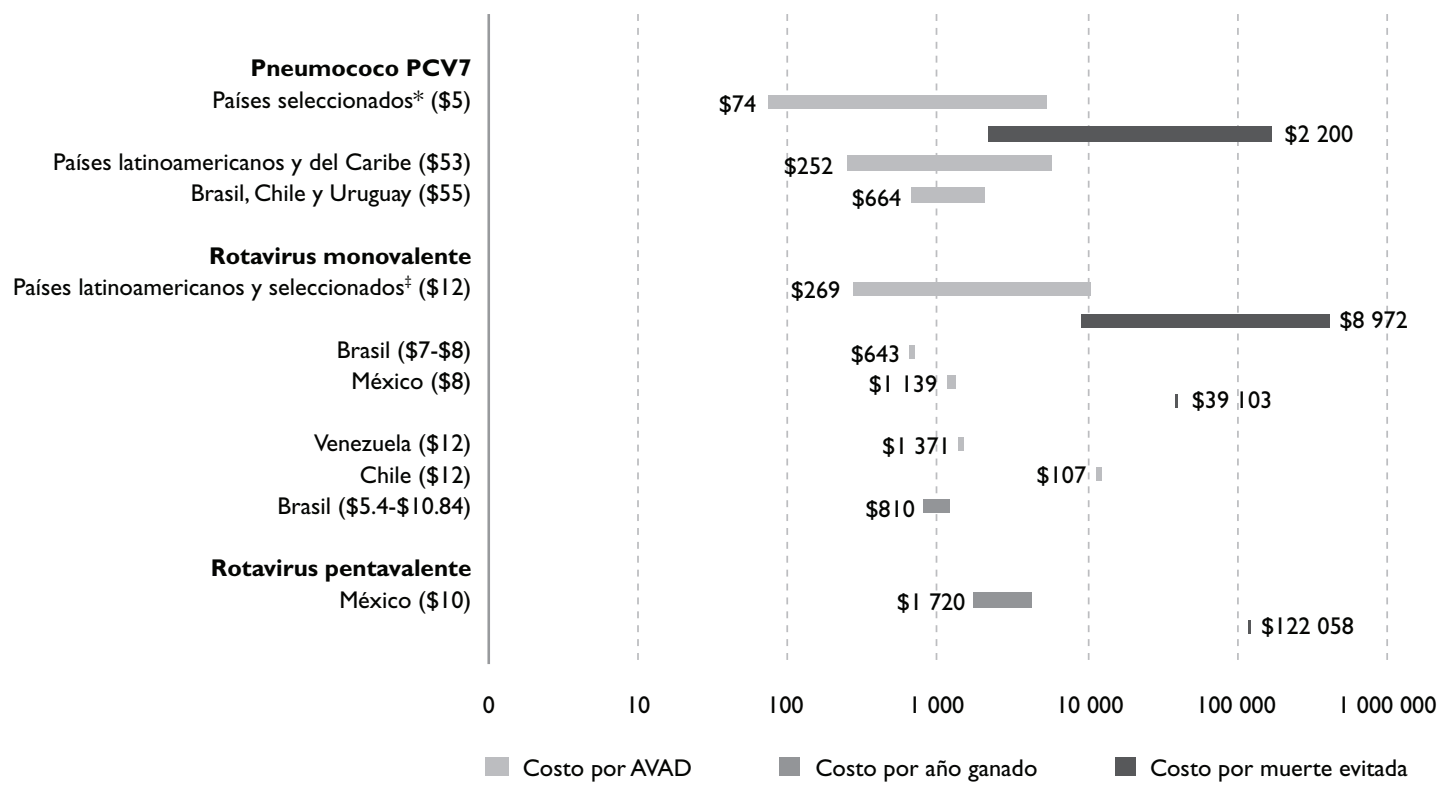

Notas:

Entre paréntesis se presentan los precios por dosis.

Para fines visuales, la gráfica se presenta en escala logarítmica.

* Considera 72 países seleccionados por la Alianza Mundial para Vacunas e Imnunización.

${ }^{\ddagger}$ Argentina, Brasil, Chile, República Dominicana, Honduras, México, Panamá y Venezuela.

Fuente: Elaboración propia a partir de la revisión de la literatura. Cuernavaca, Morelos, México.

Figura 4. Costo-efectividad de inTERVEnciones en VACunación

macroeconómicas de los países en Mesoamérica (PIB per cápita en un rango de mil a $\$ 10000),{ }^{46}$ la expansión e implementación de estas intervenciones en nutrición y en salud materno-infantil podrían considerarse como altamente costo-efectivas. Con resultados de costoefectividad generalmente mayores a los presentados por las intervenciones en nutrición y en salud maternoinfantil, puede decirse que las intervenciones en dengue y paludismo también resultan altamente costo-efectivas de acuerdo con los criterios de la Comisión de Macroeconomía y Salud de la OMS.

De acuerdo con los resultados presentados para la vacunación con la vacuna heptavelente contra neumococo, todos los rangos rebasan una vez el PIB per cápita por AVAD evitado para la mayoría de los países centroamericanos. Lo mismo sucede para dos de los cinco rangos para el caso de la vacuna monovalente contra rotavirus y para la estimación de costo por años de vida ganado de la vacuna pentavalente contra rotavirus. Cabe mencionar que, desde las fechas de publicación de los resultados de costo-efectividad de estas dos vacunas objeto de este análisis, los precios por dosis han venido en descenso, principalmente para los países de Latinoamérica que compran por medio del fondo rotatorio de la Organización Panamericana de Salud, lo que significa que las estimaciones de costo-efectividad con estos nuevos precios deberían ser menores y, por lo tanto, cada vez más dignas de ser consideradas para su inclusión dentro de los programas nacionales de vacunación. A este respecto, es importante que se generen nuevas estimaciones que tomen en cuenta los precios vigentes de las vacunas, lo que permitiría tener mejores insumos para apoyar el proceso de toma de decisiones.

Las intervenciones en cada una de estas cuatro áreas tienen características distintas en su implementación. Mientras que ciertas intervenciones en nutrición pueden implementarse de manera individual, las llevadas a cabo en el área de enfermedades transmitidas por vectores, muchas veces son secuenciales o complementarias; por esta razón, además de los criterios de eficiencia, deberían tomarse en cuenta las interacciones entre intervenciones a la hora de programar el orden en el que son introducidas o ampliadas. 


\section{Conclusiones}

Una de las conclusiones que se desprende del proceso de búsqueda de evidencia en esta investigación es que la información de costo-efectividad en el ámbito mundial es escasa para la mayoría de las intervenciones y casi inexistente en contextos de países mesoamericanos. Este hecho es relevante toda vez que las estimaciones de costo-efectividad de intervenciones en salud dependen en gran medida de situaciones locales, que incluyen el tamaño del problema de salud en ausencia de la intervención, la capacidad local para implementar las intervenciones de una manera apropiada, la aceptabilidad social de las intervenciones y la estructura local de costos. Por esta razón, las estimaciones aquí presentadas deben tomarse con cautela a la luz de las circunstancias locales.

Finalmente, como parte del proceso de planeación estratégica previo a la implementación o expansión de intervenciones en la región de Mesoamérica, es importante considerar actividades para la generación de información de costos y costo-efectividad de las intervenciones implementadas, para así poder optimizar los paquetes de intervenciones ofrecidos en el futuro en las cuatro áreas y, al mismo tiempo, generar conocimiento que pueda ser útil para la toma de decisiones en otros países o regiones.

\section{Agradecimientos}

Queremos agradecer a los expertos responsables de la elaboración del plan maestro de la IMS en las áreas de nutrición infantil, salud materno-infantil y reproductiva, vacunación, dengue y paludismo, por su orientación, retroalimentación y apoyo en la realización de este trabajo. Se agradece también el apoyo de Araceli Martínez Pérez, Josué del Ángel Martínez y Norma Padilla Eguiluz, quienes apoyaron de manera fundamental las diversas actividades de este estudio. Finalmente, queremos agradecer el financiamiento para este proyecto, proporcionado por la Fundación Bill y Melinda Gates vía el Public Health Institute, al hacer posible la realización de este estudio que genera conocimiento para la región de Mesoamérica.

Declaración de conflicto de intereses: Los autores declararon no tener conflicto de intereses.

\section{Referencias}

I. Proyecto Mesoamérica [sitio de internet]. Sistema Mesoamericano de Salud Pública (SMSP). El Salvador: SMSP, 2010. [consultado: 2010 agosto]. Disponible en: http://portal2.sre.gob.mx/mesoamerica/index. php?option=com_content\&task=view\&id=57\&ltemid $=45$
2. Instituto Nacional de Salud Pública (INSP), Grupo Técnico de Nutrición (GTN). México: Instituto Nacional de Salud Pública, Iniciativa Mesoamericana de Salud, 2009.

3. Instituto Nacional de Salud Pública (INSP), Grupo Técnico de Salud Materna y Reproductiva (GTSMR). Reproductive, Maternal and Neonatal Health Master Plan. Iniciativa Mesoamericana de Salud, 2009.

4. World Health Organization (WHO), Choosing Interventions that are Cost Effective (CHOICE) [sitio de internet]. Geneva:WHO, 2009. [consultado: 2010 agosto]. Disponible en: http://www.who.int/choice/en/ 5. Cairncross S,Valdmanis V.Water Supply, Sanitation,and Hygiene Promotion. En: Jamison DT, Breman JG, Measham AR, Alleyne G, Claeson M, Evans DB, et al. eds. Disease control priorities in developing countries. 2a. edición. Nueva York: Oxford University Press, 2006;41:77I-792. 6. Hoddinot J, Bassett L. Conditional Cash Transfer programs and Nutrition in Latin America:Assessment of impacts and strategies for improvement. Hunger-Free Latin America and the Caribbean Initiative, Working Paper 9. FAO; 2009.

7. Horton S, Sanghvi T, Phillips M, Fiedler J, Perez-Escamilla R, Lutter C, et al. Breastfeeding promotion and priority setting in health. Health Policy Plan 1996; I (2): I56-I68.

8. Caulfield LE, Richard SA, Rivera JA, Musgrove P, Black RE. Stunting, Wasting, and Micronutrient Deficiency Disorders. In: Jamison DT, Breman JG, Measham AR, Alleyne G, Claeson M, Evans DB, et al. eds. Disease control priorities in developing countries. 2a edición. Nueva York: Oxford University Press, 2006;28:55I-567.

9. Fiedler JL.A Cost Analysis of the Honduras Community-Based Integrated Child Care Program (Atención Integral a la Niñez-Comunitaria, AIN-C).Washington:World Bank, 2003.

I0. Llanos A, Hertrampf E, Cortes F, Pardo A, Grosse SD, Uauy R. Costeffectiveness of a folic acid fortification program in Chile. Health Policy 2007;83(2-3):295-303.

II. Baltussen R, Knai C, Sharan M. Iron fortification and iron supplementation are cost-effective interventions to reduce iron deficiency in four subregions of the world. J Nutr 2004;134(I0):2678-2684.

12. Horton S, Sheekar M, McDonald C, Mahal A, Brooks JK. Scaling UP Nutrition: What will it cost? Washington: The World Bank; 2010. 13. Sharieff W, Horton SE, Zlotkin S. Economic gains of a home fortification program: evaluation of "Sprinkles" from the provider's perspective. Can J Public Health 2006;97(I):20-23.

14. Graham WJ, Cairns J, Bhattacharya S, Bullough CHW, Quayyum Z, Rogo K. Maternal and Perinatal Conditions. En: Jamison DT, Breman JG, Measham AR,Alleyne G, Claeson M, Evans DB, et al, eds. Disease Control Priorities in Developing Countries. 2a edición. Nueva York: Oxford University Press, 2006:26.

15. Haws RA, Thomas AL, Bhutta ZA, Darmstadt GL. Impact of packaged interventions on neonatal health: a review of the evidence. Health Policy Plan 2007;22(4): 193-2I5.

16. Darmstadt G, Bhutta Z, Cousens S,Adam T,Walker N, de Bernis L. Evidence-based, cost-effective interventions: how many newborn babies can we save? Lancet 2005;365(9463):977-988.

17. Jowett M. Cost-effective safe motherhood interventions in low-income countries: a review. Centre for Health Economics, University of York, 2000. 18. Suaya J, Shepard D, Chang MS, Caram M, Hoyer S, Socheat D, et al. Cost-effectiveness of annual targeted larviciding campaigns in Cambodia against the dengue vector Aedes aegypti. Trop Med Int Health 2007;12(9): 1026-1036

19. Shepard DS, Suaya JA, Halstead SB, Nathan MB, Gubler DJ, Mahoney $\mathrm{RT}$, et al. Cost-effectiveness of a pediatric dengue vaccine.Vaccine 2004;22(9-10): 1275-I 280.

20.Akhavan D, Musgrove P,Abrantes A, d'A Gusmão R. Cost-effective malaria control in Brazil. Cost-effectiveness of a Malaria Control Program in the Amazon Basin of Brazil, 1988-1996. Soc Sci Med 1999;49(I0): 1385-1399. 2I. Hanson K, Kikumbih N,Armstrong-Schellenberg J, Mponda H, Nathan $\mathrm{R}$, Lake $\mathrm{S}$, et al. Cost-effectiveness of social marketing of insecticide- 
treated nets for malaria control in the United Republic of Tanzania. Bull World Health Organ 2003;8I (4):269-276.

22. Mueller DH, Wiseman V, Bakusa D, Morgah K, Daré A, Tchamdja P. Cost-effectiveness analysis of insecticide-treated net distribution as part of the Togo Integrated Child Health Campaign. Malar J 2008 29;7:73. 23. Yukich JO, Zerom M, Ghebremeskel T, Tediosi F, Lengeler C. Costs and cost-effectiveness of vector control in Eritrea using insecticide-treated bed nets. Malar J 2009 30;8:5I.

24. Hutton G, Schellenberg D, Tediosi F, Macete E, Kahigwa E, Sigauque $B$, et al. Cost-effectiveness of malaria intermittent preventive treatment in infants (IPTi) in Mozambique and the United Republic of Tanzania. Bull World Health Organ 2009;87(2):I23-I29.

25. Utzinger J, Tozan Y, Singer BH. Efficacy and cost-effectiveness of environmental management for malaria control. Trop Med Int Health 200I;6(9):677-687.

26. Yukich JO, Lengeler C, Tediosi F, Brown N, Mulligan JA, Chavasse D, et al. Costs and consequences of large-scale vector control for malaria. Malar J 2008 I7;7:258.

27. Goodman CA, Mnzava AE, Dlamini SS, Sharp BL, Mthembu DJ, Gumede JK. Comparison of the cost and cost-effectiveness of insecticide-treated bednets and residual house-spraying in KwaZulu-Natal, South Africa. Trop Med Int Health 200I;6(4):280-295.

28. Binka FN, Mensah OA, Mills A. The cost-effectiveness of permethrin impregnated bednets in preventing child mortality in Kassena-Nankana district of Northern Ghana. Health Policy 1997;4I(3):229-239.

29. Sinha A, Constenla D, Valencia JE, O'Loughlin R, Gomez E, de la Hoz F, et al. Cost-effectiveness of pneumococcal conjugate vaccination in Latin America and the Caribbean: a regional analysis. Rev Panam Salud Publica 2008;24:304-3/3.

30. Constenla DO. Economic impact of pneumococcal conjugate vaccination in Brazil, Chile, and Uruguay. Rev Panam Salud Publica 2008;24:101-112

3I. Sinha A, Levine O, Knoll MD, Muhib F, Lieu TA. Cost-effectiveness of pneumococcal conjugate vaccination in the prevention of child mortality: an international economic analysis. Lancet 2007;369:389-396.

32. Constenla D, Velazquez FR, Rheingans RD, Antil L, Cervantes Y. Economic impact of a rotavirus vaccination program in Mexico. Rev Panam Salud Publica 2009;25:48I-490.

33. Constenla DO, Linhares AC, Rheingans RD,Antil LR, Waldman EA, da Silva LJ. Economic impact of a rotavirus vaccine in Brazil.J Health Popul Nutr 2008;26:388-396.
34. de Soarez PC,Valentim J, Sartori AM, Novaes HM. Cost-effectiveness analysis of routine rotavirus vaccination in Brazil. Rev Panam Salud Publica 2008;23:221-230.

35. Rheingans RD, Constenla D, Antil L, Innis BL, Breuer T. Potential cost-effectiveness of vaccination for rotavirus gastroenteritis in eight Latin American and Caribbean countries. Rev Panam Salud Publica 2007;21:205-216.

36. Constenla D, O'Ryan M, Navarrete MS, Antil L, Rheingans RD. Potential cost effectiveness of a rotavirus vaccine in Chile. Rev Med Chil 2006; 134:679-688

37. Constenla D, Perez-Schael I, Rheingans RD,Antil L, Salas H, Yarzabal JP.Assessment of the economic impact of the antiretroviral vaccine in Venezuela. Rev Panam Salud Publica 2006;20:213-222.

38. Valencia A, Bertozzi S, Gutierrez JP, Itzler R. Cost-effectiveness of introducing rotavirus vaccine in developing countries: the case of Mexico. BMC Infect Dis 2008;8: 103.

39. Waters HR, Penny ME, Creed-Kanashiro HM, Robert RC, Narro $R$,Willis J, et al.The cost-effectiveness of a child nutrition education programme in Peru. Health Policy Plan 2006;2I (4):257-264.

40. Gross R, Gross U, Lechtig A, Lopez de Romana D.We know much about what to do but little about how to do it: experiences with a weekly multimicronutrient supplementation campaign. Food Nutr Bull 2006;27(4 Suppl Peru):SI II-I I4.

4I. Postma MJ, Londeman J,Veenstra M, de Walle HE, de Jong-van den Berg LT. Cost-effectiveness of periconceptional supplementation of folic acid. Pharm World Sci 2002;24(I):8-II.

42. Adam T, Lim SS, Mehta S, Bhutta ZA, Fogstad H, Mathai M, et al.Achieving the millennium development goals for health. BM] 2005;33I (7525): I I 07.

43. Griffiths U,Wolfson L, Quddus A, Younus M, Hafiz R. Incremental cost-effectiveness of supplementary immunization activities to prevent neonatal tetanus in Pakistan. Bull World Health Organ 2004;82(9):643-65I. 44. Trussell J, Lalla AM, Doan QV, Reyes E, Pinto L, Gricor J, et al. CostEffectiveness of Contraceptives in the United States. Contraception 2009;79|):5-14.

45. Evans DB, Adam T,Tan-Torres ET, Lim SS, Cassels A, Evans TG.Achieving the millennium development goals for health. BMJ 2005;33 I (7525): I I33-I I36.

46. The World Bank. GDP per capita (current US\$). [consultado 2010 agosto]. Disponible en: http://data.worldbank.org/indicator/NY.GDP. MKTP.CD. 